Old Dominion University

ODU Digital Commons

2010

\title{
Operation and Application of a Regional High-Frequency Radar Network in the Mid-Atlantic Bight
}

\author{
Hugh Roarty \\ Scott Glenn \\ Josh Kohut \\ Donglai Gong \\ Ethan Handel
}

See next page for additional authors

Follow this and additional works at: https://digitalcommons.odu.edu/ccpo_pubs

Part of the Oceanography Commons

\section{Original Publication Citation}

Roarty, H., Glenn, S., Kohut, J., Donglai, G., Handel, E., Rivera, E., . . Seim, H. (2010). Operation and application of a regional high-frequency radar network in the Mid-Atlantic Bight. Marine Technology Society Journal, 44(6), 133-145. doi:10.4031/MTSJ.44.6.5

This Article is brought to you for free and open access by the Center for Coastal Physical Oceanography at ODU Digital Commons. It has been accepted for inclusion in CCPO Publications by an authorized administrator of ODU Digital Commons. For more information, please contact digitalcommons@odu.edu. 


\section{Authors}

Hugh Roarty, Scott Glenn, Josh Kohut, Donglai Gong, Ethan Handel, Erick Rivera, Teresa Garner, Larry Atkinson, Wendell Brown, Chris Jakubiak, Mike Muglia, Sara Haines, and Harvey Seim 


\section{Operation and Application of a Regional High-Frequency Radar Network in the Mid-Atlantic Bight}

A UTHORS

Hugh Roarty

Scott Glenn

Josh Kohut

Donglai Gong

Ethan Handel

Erick Rivera

Coastal Ocean Observation

Laboratory, Rutgers University

Teresa Garner

Larry Atkinson

Center for Coastal

Physical Oceanography,

Old Dominion University

Wendell Brown

Chris Jakubiak

School for Marine Science

and Technology, University

of Massachusetts Dartmouth

Mike Muglia

Sara Haines

Harvey Seim

University of North Carolina

at Chapel Hill

\section{Introduction}

nsights arising from a deeper understanding of surface currents can be valuable when one seeks to characterize and quantify the transport of plankton and anthropogenic material in the coastal ocean. The MidAtlantic Bight High-Frequency (HF) Radar Network, which is comprised of 13 long-range sites, two mediumrange sites, and 12 standard-range sites, is operated as part of the Inte-

\section{A B S T R A C T}

The Mid-Atlantic Regional Coastal Ocean Observing System (MARCO0S) HighFrequency Radar Network, which comprises 13 long-range sites, 2 medium-range sites, and 12 standard-range sites, is operated as part of the Integrated Ocean Observing System. This regional implementation of the network has been operational for 2 years and has matured to the point where the radars provide consistent coverage from Cape Cod to Cape Hatteras. A concerted effort was made in the MARCOOS project to increase the resiliency of the radar stations from the elements, power issues, and other issues that can disable the hardware of the system. The quality control and assurance activities in the Mid-Atlantic Bight have been guided by the needs of the Coast Guard Search and Rescue Office. As of May 4, 2009, these quality-controlled MARCOOS High-Frequency Radar totals are being served through the Coast Guard's Environmental Data Server to the Coast Guard Search and Rescue Optimal Planning System. In addition to the service to U.S. Coast Guard Search and Rescue Operations, these data support water quality, physical oceanographic, and fisheries research throughout the Mid-Atlantic Bight.

Keywords: HF radar, Networks, Ocean currents, Remote sensing

grated Ocean Observing System. This regional implementation of the network has been operational for 2 years and has matured to the point where the radars provide consistent coverage from Cape Cod to Cape Hatteras. This is based on a significant effort to ensure hardware and software resiliency, quality control, and quality assurance (QA). Spatial coverage has been observed to vary on daily and seasonal scales because of ionospheric interference at the lower end of the HF radio spectrum and variable sea state conditions (Liu et al., 2010). Through a partnership with the U.S. Coast Guard Research and Development Center and Office of Search and Rescue, Mid-Atlantic Regional Coastal Ocean Observing System (MARCOOS) partners have worked to advance this network to provide consistent and accurate surface current information to search and rescue operations. As a result of this partnership, surface currents are automatically delivered to the Coast Guard with improved quality control of the data and dissemination of typical current patterns and anomalous conditions to search and rescue personnel. In turn, the improved system quality has supported basic oceanographic research, water quality applications, numerical and statistical model assimilation, and environmental monitoring associated with offshore energy development throughout the Mid-Atlantic Bight. In this article, we will describe the network as it operates today, giving special 
attention to the resiliency of the hardware and the flow of data from the sites on shore to regional and national data networks (Section 2). In Section 3, we describe the data processing at the radial and total level. The quality control, assurance, and data evaluation are summarized in Section 4. Finally in Section 5, we present some of the regional applications of the network.

\section{The Network}

\subsection{Hardware}

The MARCOOS HF Radar Network consists of 27 SeaSondetype radars, 13 of which are long range (Figure 1), 12 of which are standard range, and 2 of which are medium range. Table 1 provides the typical characteristics of the different types of systems. Each site consists of two categories of hardware: the radar

\section{FIGURE 1}

Location of the long-range HF radar locations (circles) within the MARCOOS region with four-letter site code next to station location.

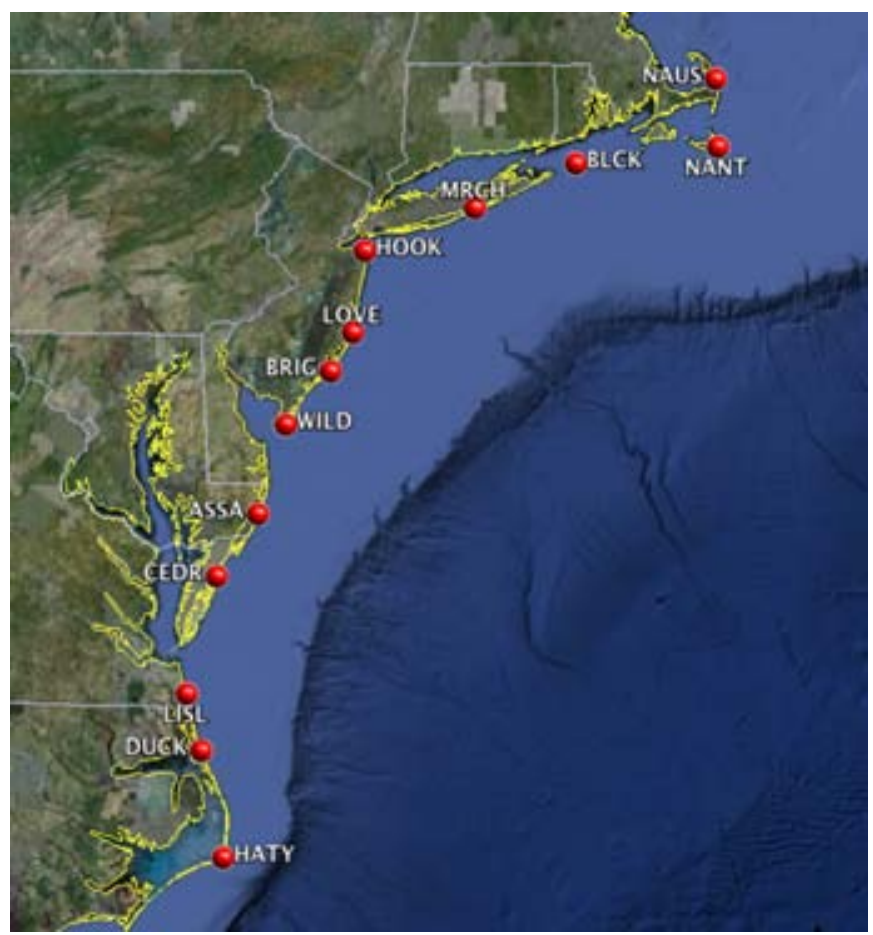

\section{TABLE 1}

equipment purchased directly from CODAR Ocean Sensors and the ancillary site-specific hardware required for communications, power, backup power, temperature control, weather proofing, security, and antenna foundations.

A typical HF radar system purchased from CODAR Ocean Sensors includes a transmitter, receiver, transmit antenna, receive antenna, Apple Computer, cabling, and a GPS antenna. In addition, a CODAR Ocean Sensors transponder unit is required for site calibration. A few vendor hardware op-
Typical characteristics of long-, medium-, and standard-range HF radar systems.

\begin{tabular}{l|c|c|c}
\hline System Type & Radio Frequency $\mathbf{( M H z )}$ & Range $\mathbf{( k m )}$ & Resolution $\mathbf{( k m )}$ \\
\hline Long range & $4-6$ & 200 & 6.0 \\
\hline Medium range & $12-14$ & 90 & 3.0 \\
\hline Standard range & $24-26$ & 40 & 1.5 \\
\hline
\end{tabular}

tions are available such as different computer types (laptop, desktop, or compact) and combined transmit and receive antennas for all but the longrange systems. The transmit and receive units are rack-mounted units approximately the size of a home stereo receiver. Transmit antenna sizes vary with the frequency of the system, from

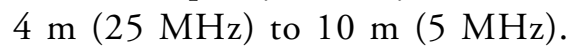
Three RG-58 cables connect the SeaSonde Receiver to the receive antenna. A single RG-8 cable connects the SeaSonde Transmitter to the transmit antenna. Typical cable runs are up to $100 \mathrm{~m}$ to each antenna.

The site-specific hardware varies widely on the basis of site requirements and the operator's experience. Typical considerations include power (both primary and backup), communications, climate control, security and vandalism, consideration for erosion, and antenna foundations. Communication for data transmission in near real time can be done with a phone line, but higher bandwidths are preferable for remote computer control and trouble shooting applications where available. A secondary communication option is encouraged. The number of sites with two lines of communication increased from three to nine from 2007 to 2009.

Communications sources in our region include cable Internet, DSL, telephone, satellite Internet, cellular modems, radio frequency communications, and short-range wireless 


\section{TABLE 2}

Primary communication modes for network in years 2007 through 2009.

\begin{tabular}{l|c|c|c}
\hline & $\mathbf{2 0 0 7}$ & $\mathbf{2 0 0 8}$ & $\mathbf{2 0 0 9}$ \\
\hline Phone & 8 & 2 & 0 \\
\hline Cell modem & 8 & 9 & 13 \\
\hline DSL & 0 & 5 & 5 \\
\hline Cable modem & 9 & 9 & 9 \\
\hline Number of sites with two lines of communication & 3 & 9 & 9 \\
\hline
\end{tabular}

for automated toggling of components that are not functioning properly.

A concerted effort was made in the MARCOOS project to increase the resiliency of the radar stations from the elements, power issues, and other issues that can disable a system. The optimal configuration of the shore station is shown in Figure 2. The site uses a TrippLite UPS with optional Web card as recommended in the Southern California Coastal Ocean Observing telemetry from an Internet source. Communication preference is given to the most robust connection and to a redundant communication source. Table 2 provides a history of the primary communication methods used in the network. The phone line, which has been phased out as the primary communication method, has been kept at all sites to serve as a secondary line of communication. This line can be used for data transfer when the primary method fails and can also be used to control power cycling devices that restore the primary communication method in the event of an outage. Having a fixed Internet Protocol address at a site allows for more robust diagnostic capabilities.

All sites in the network have access to the power grid. All sites use of an uninterrupted power supply (UPS) to provide a "cleaner" source of power as well as to eliminate power loss during outages of less than $30 \mathrm{~min}$. In addition, some sites use backup power such as a propane generator and transfer switch to eliminate outages during inclement weather like tropical storms to maintain the data time series through these significant oceanographic events. Remote control devices such as the Powerstone, iBoot, and Web Power Switch are used for toggling power to separate components

\section{FIGURE 2}

Power (top) and communication (below) configuration for resilient HF radar station.

\section{Loveladies Power Configuration}

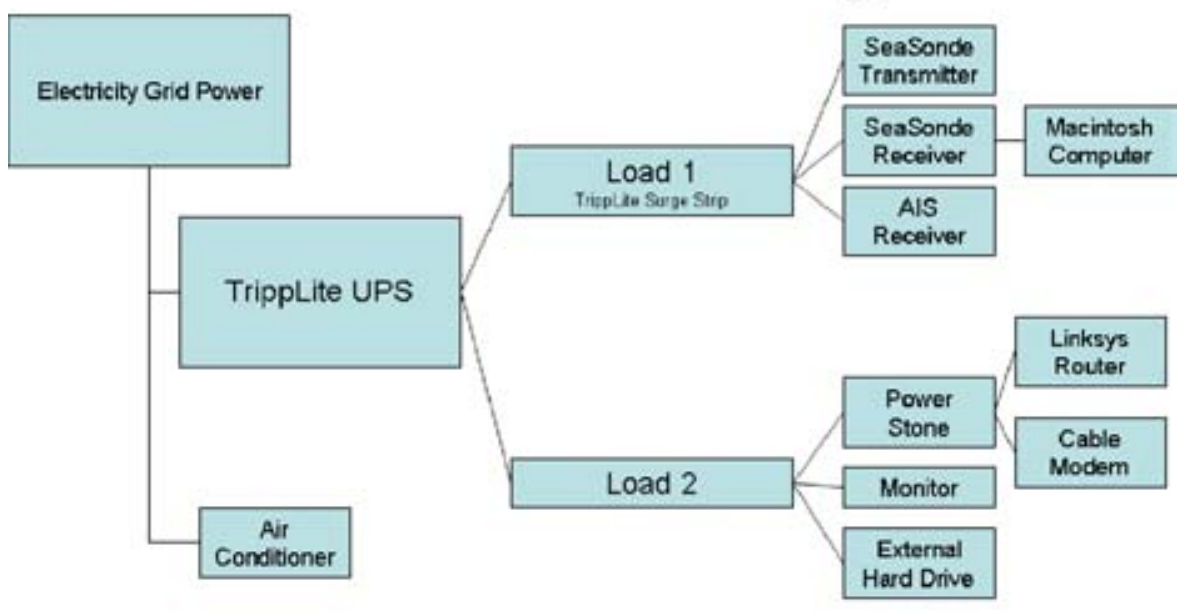

Loveladies Communication Configuration

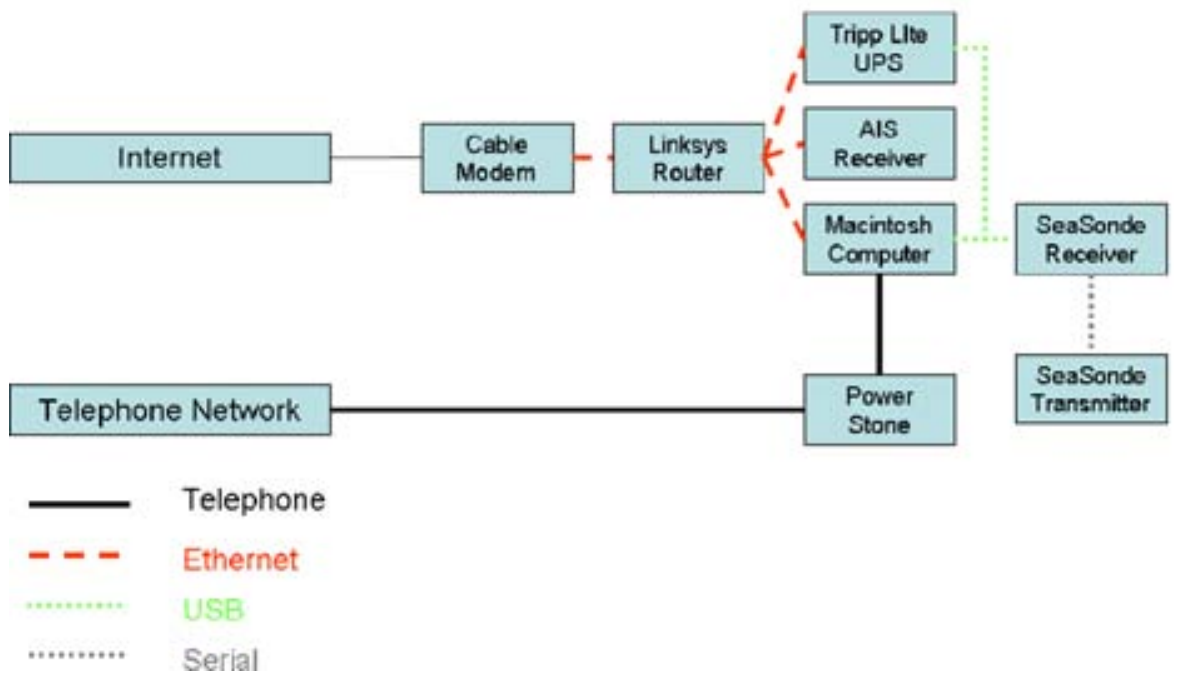


System Best Practices Document. This UPS has two power loads that can cycle power remotely to the devices on each load. This Web card will log power interruptions, send notifications of the power interruption, and allow for remote cycling of power to individual components of the system. Electrical power to the router and cable modem is directed through the Power Stone so that if either device freezes, the secondary telephone line can be used to cycle power to these components. Environmental events in our region like coastal storms and associated lightning can cause prolonged outages because of hardware damage. To counter one of the more common events in our region, lightning, we are testing a protection device that is designed to protect the transmitter and receiver from direct lightning strikes to the antennas. In addition, we are continually monitoring the local environments of the antennas to ensure that the sites are operating optimally. Since the 2007, three sites were relocated with the specific objective to improve the data quality provided by the site. One site had poor coverage because of a long cable run $(150 \mathrm{~m})$, so this site was moved so the cable run would be the standard length of $100 \mathrm{~m}$. The other two sites that were moved had distorted antenna patterns because of the presence of a large structures in the vicinity of the receive antenna. These sites were moved to a "clear environment" (Kohut and Glenn, 2003) free of known conductors. All moves resulted in improved radial coverage and hence improved total coverage.

\subsection{Data Flow}

Each site described above collects hourly measurements of the radial surface currents and wave conditions within a footprint local to the antenna.
For surface currents this footprint can be as large as $200 \mathrm{~km}$ from the site with $6-\mathrm{km}$ resolution for the $5-\mathrm{MHz}$ systems to higher resolution $25 \mathrm{MHz}$ systems that stretch $50 \mathrm{~km}$ with a spatial resolution of $1 \mathrm{~km}$. These data are first collected at the local central computer sites for each of the eight operators in the region (Rutgers University, University of Massachusetts, University of Rhode Island, University of Connecticut, Stevens Institute of Technology, University of Delaware, Old Dominion University, and University of North Carolina). The radial data are then aggregated at Rutgers as part of the National HF Radar data server supported by the National Oceanic and Atmospheric Administration. The radial data from the long-range sites is combined into total vectors on a low-resolution $6-\mathrm{km}$ regional scale grid that covers coastal waters from Cape Cod to Cape Hatteras. The total vector fields are made available via Open-source Project for a Network Data Access Protocol (OPeNDAP) for assimilation into the University of Connecticut's Short Term Prediction
System (STPS) and an ensemble of three dynamical forecast models run by Rutgers, Stevens Institute of Technology, and University of Massachusetts, Dartmouth. The total vector fields and statistical forecasts are then transferred to the US Coast Guards Environmental Data Server (EDS), which is managed by Applied Science Associates. Once in EDS, the data and the forecasts underwent a year-long test phase within the Coast Guard's new Search and Rescue Optimal Planning System (SAROPS). This data flow is summarized in Figure 3. After the test phase at the Coast Guard office of Search and Rescue, the accepted data and model forecasts are available in the field offices that have access to SAROPS. In May 2009, the data met that criteria and became an operational data stream of the U.S. Coast Guard Office of Search and Rescue.

\subsection{Operation and Maintenance}

At the beginning of the MARCOOS effort, several steps were taken to standardize the practices of the individual operators and subregional networks

\section{FIGURE 3}

Schematic showing the data flow from individual radar sites to the Coast Guard SAROPS.

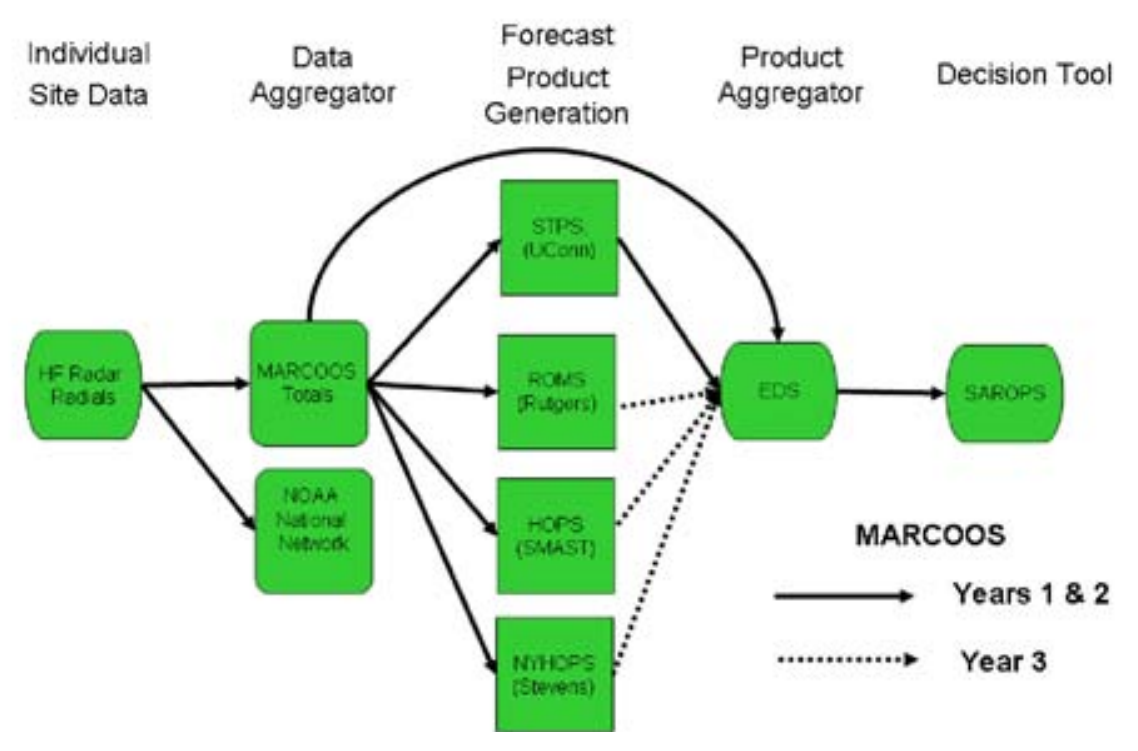


already in place. An operator working group was formed, and conference calls were held every 2 weeks to discuss the progress of the project. The existing sites in the region were inventoried, and an online database of hardware and software was developed. On the basis of this inventory, software versions were standardized throughout the network. All sites running CODAR software were updated to Release 5 Update 3. The current version of CODAR software is Release 6 Update 2, and all operators were encouraged to upgrade to the latest release. Three QA settings were implemented on all sites as of April 1, 2008:

- The "Minimum Radial Vector Filter" was set to 2 . This is the second parameter on line 1 of the AnalysisOptions.txt file

- The "Radial Factor Above Noise" was set to 5 . This is the second parameter on line 15 of the Header. txt file

- The measured antenna phases were checked against those set in the SeaSonde Radial Setup application. If there was a difference of more than $15^{\circ}$, the set phases were changed to match the measured phases.

These settings were established on the basis of data evaluation (Section 4 of this article) to ensure real-time QA of the radial data. Computer scripts to monitor these and other site settings and data quality were developed, and a network-wide diagnostic monitoring Website was developed and installed. In addition to the Websites, an e-mail is sent daily to the operators reporting on the radial file size and latency of each radial file on the $\mathrm{Na}$ tional Network.

All HF radar sites in the MidAtlantic were set up to report their data to the National Oceanic and Atmospheric Administration National
Network Server at Rutgers. HF radar operations were sustained at a rate consistent with Phase 2 of the MidAtlantic HF Radar Consortium's three-phase implementation plan. Phase 2 includes three full-time HF radar technicians distributed across the northern, central, and southern subregions of the Mid-Atlantic with a part time regional coordinator managing the technicians and network. A week-long advanced training session was held in February 2008. The three full-time technicians as well as technicians from seven of the eight operators in the region attended this training. At this meeting, it was decided that the regional HF radar network would adopt a distributed technician approach, with one operator responsible for the systems in each of the three regions (north, central, and south). This work force was able to achieve an $89 \%$ operating time for the long-range systems from December 1, 2008, to November 30, 2009 (Table 3).

\section{Data Processing 3.1. Radial Processing}

A suite of CODAR software programs processes the received radar signals to generate the hourly radial current files at each site. Further processing combines the radials from two or more sites to produce total current velocity vector maps. The radar system determines wave speed by measuring the Doppler shift between a transmitted radio signal and its return signal reflected off of ocean waves (Barrick et al., 1977). The CODAR radar software empirically isolates the strongest sea echo returns because of Bragg scattering and uses these to calculate radial current velocity. According to the Bragg principle, these strong reflections, referred to as firstorder sea echo, come from waves of a

\section{TABLE 3}

Radar operational time as a percentage of the time from December 1, 2008, till November 30, 2009.

\begin{tabular}{l|c}
\hline $\begin{array}{l}\text { Four-Letter } \\
\text { Site Code }\end{array}$ & $\begin{array}{l}\text { Operating } \\
\text { Time (\% year) }\end{array}$ \\
\hline NAUS & 93 \\
\hline NANT & 92 \\
\hline BLCK & 90 \\
\hline MRCH & 97 \\
\hline HOOK & 98 \\
\hline LOVE & 98 \\
\hline BRIG & 99 \\
\hline WILD & 69 \\
\hline ASSA & 92 \\
\hline CEDR & 59 \\
\hline LISL & 71 \\
\hline DUCK & 100 \\
\hline HATY & 100 \\
\hline Network average & 89 \\
\hline
\end{tabular}

known wavelength, half that of the transmitted electromagnetic wave (Crombie, 1955). The Doppler shift of these waves in the absence of ocean current is proportional to the phase velocity given by the deep water dispersion equation for gravity waves. The difference between the observed first-order Doppler shift and the shift due to wave speed represents the speed of the surface current underlying the wave (Lipa and Barrick, 1983).

Each 5-MHz Mid-Atlantic radar site measures these one-dimensional radial current velocities, directed toward or away from the antenna, in 6-km-range bins and in $5^{\circ}$ directional bins. To do this, two spectral analyses are performed within the software. The first separates the incoming raw voltage time series into different range bins, whereas the second transforms the range-dependent time series, 
resulting from the first into Doppler spectra binned by range. These spectra contain the first-order Bragg scatter used to extract the radial currents. Because SeaSondes are direction-finding systems, the bearing of the radial vectors is determined from the signal received from three separate antennas using the MUSIC algorithm (Lipa et al., 2006). Using a transponder, the angular-dependent response of each antenna can be incorporated into to the processing as a way to calibrate the system for distortions to the antenna pattern (Barrick and Lipa, 1986; Kohut and Glenn, 2003). The operational time from each of the long-range sites is given in Table 3 .

\subsection{Total Vector Processing}

During the extension of the network to the regional footprint, we have processed radials to totals using two algorithms, unweighted least squares (UWLS; Lipa and Barrick, 1983) and Optimal Interpolation (Kim et al., 2008). The first approach merges radial vectors located within a search radius around each grid point using a UWLS fitting method (Lipa and Barrick, 1983). The CODAR combine software uses this method as well as the community Matlab toolbox, HFR_Progs. The regional radialto-total processing is accomplished within Matlab. In the Mid-Atlantic, the search radius for the UWLS method is $10 \mathrm{~km}$, and the spacing for the grid is $8 \mathrm{~km}$. A minimum of three radials from at least two sites are required to calculate a total and the geometric dilution of precision uncertainty estimate for the vector must be less than 1.25 to pass quality control checks. The second technique for computing totals uses optimal interpolation (OI) adaptation developed by Kim et al. (2008). For this method, we used an asymmetric search area stretch in the along-isobath direction and consistent with the length scales of the currents in the region. For QA, we require that both the $\mathrm{u}$ and $\mathrm{v}$ component uncertainty be less than $60 \%$ the expected variance. The MARCOOS real-time processing scripts output results using both methods. Through the evaluation discussed later in this article, the total vector product delivered operationally is based on the OI.

\section{Surface Current Evaluation}

\subsection{Quality Control and QA}

The quality control and assurance activities in the Mid-Atlantic Bight have been guided by the needs of the Coast Guard Search and Rescue Office. QA is a set of procedures done to instrumentation and a system of processing that ensure quality and measure uncertainties. Quality control is the activity of testing the data against defined standards or measured uncertainties to ensure quality. QA includes following the manufacture's installation guidelines of hardware and sighting of systems to avoid known interference. Toward this end and because all the systems in the region are CODAR systems, MARCOOS developed a set of recommendations to follow in hardware setup (Roarty, 2009) and radial vector processing software (Kohut, 2008) that ensures that all new sites are configured properly and in a consistent manor across the region. QA also includes understanding the environment of HF distortions and minimizing interferences to the return signal, which can vary dramatically from site to site. Each site in the region ensures their radial quality by measuring the HFR receive antenna pattern once it is located and set up (Kohut, 2008) and then using the measured pattern in the software. This measured pattern serves to calibrate the software with the actual antennas response in the field. In addition, a collaborative effort between all sites using the same frequency was conducted to ensure no one site causes contamination at a neighboring site or sites. This is performed using the GPS synchronization capability (Barrick et al., 2001) of the SeaSonde. Finally, each group monitors a site's health, raw spectra, and radial output by manual or automated means and ensures that the site is operating within its hardware specifications and that data are delivered in a timely fashion. As part of the MARCOOS effort and the delivery of regional and subregional HF radar data, there is a need to define the uncertainty bounds of the data for effective utility in SAROPS (Roarty, 2009). The UWLS geometric dilution of precision uncertainty estimate must be less than 1.25 to pass quality control checks (see Software Section). With OI, normalized velocity uncertainty of velocity components is determined. A threshold of $60 \%$ of the error variance for either the $\mathrm{u}$ or $\mathrm{v}$ component was chosen to remove any grid points in real-time data on the basis of this uncertainty threshold to maximize data coverage while preserving data quality (Kohut et al., 2009).

\subsection{Integration with SAROPS}

Quality-controlled MARCOOS HF Radar totals are being served through the Coast Guard's EDS to the Coast Guard SAROPS as of May 4, 2009. Before the introduction of the HF radar product to the Coast Guard decision tool, an extensive validation and evaluation was done. A focus of this evaluation was to determine the most accurate algorithm for combining radial vectors into totals that 
would provide consistent accurate coverage.

Using a test period in the winter to spring of 2007 , totals generated with both the existing UWLS and the new OI algorithms were compared with four moored acoustic Doppler current profilers (ADCPs) and seven surface drifters. The analysis included sensitivity to input parameters to OI, including expected variances and spatial decorrelation scales. The specific sites used include Sandy Hook, NJ (HOOK), Loveladies, NJ (LOVE), Wildwood, NJ (WILD), and Assateague, MD (ASSA). Each site was operated with the QA/QC recommendations from the regional operators and the Radiowave Operators Working Group community providing radial data to these standards.

$A D C P$ : Four ADCPs were deployed off the coast of New Jersey as part of the National Science Foundation supported Mid-Shelf Front Experiment. Three of the moorings were oriented in a cross-shelf line approximately $10 \mathrm{~km}$ apart. The shallowest mooring, deployed in $45 \mathrm{~m}$ of water, was a $300-\mathrm{kHz}$ unit. The midpoint mooring in $53 \mathrm{~m}$ of water was a $600-\mathrm{kHz}$ unit, and the offshore $600-\mathrm{kHz}$ unit was in approximately $54 \mathrm{~m}$ of water. A third $600-\mathrm{kHz}$ unit mooring deployed $11 \mathrm{~km}$ upshelf of the midpoint mooring was deployed in $50 \mathrm{~m}$ of water. All units were configured with $2-\mathrm{m}$ bins in the vertical. Sampling was configured to collect a 10-min ensemble each hour. These data were then averaged to match the sampling of the HF radar.

Drifters: The Self-Locating Data Marker Buoy (SLDMB) position data were used to evaluate the CODAR observations. The SLDMB drifters were provided by the U.S. Coast Guard. They had exceeded their shelf life for use in life saving operations but remained excellent platforms to evaluate the performance of the two combination algorithms. The drifters were drogued to 1-m depth. Throughout the deployment, velocities on the basis of two drifter positions $1 \mathrm{~h}$ apart were calculated every half hour. These surface velocity estimates were compared with the radial and total vector estimates of the long-range CODAR network off the New Jersey coast. For the total vector comparisons, the velocity average was set to match the sampling of the CODAR. The SLDMB data discussed here are from two deployments in the winter and spring of 2007. The first deployment included two drifters deployed on February 24, 2007. Since the one deployed inside the mid-shelf front spent more time within the coverage of the radar, it was used in the analysis. The second deployment began April 3, 2007, and included six drifters. Although this second group of drifters did not overlap with the ADCP deployments, the larger cluster of drifters provides an extended data set to explore the impact of spatial variability on the comparisons. All of the deployments together allow us to explore spatial dependency in the evaluation particularly close to the offshore edge of the coverage. A more thorough explanation of the evaluation is given by Kohut et al. (in preparation). An example of one ADCP and one drifter comparison is provided in Table 4.

Comparisons between both the UWLS and the OI total vector solutions showed significant agreement with the in situ measurements of both the ADCPs and the drifters. Root mean square (RMS) differences ranged from 7.5 to $11.8 \mathrm{~cm} / \mathrm{s}$ over the study period (Chapman and Graber, 1997). It is important to note that the ADCPs were deployed in a region of very good geometric coverage of the radial sites used in the total vector combination, whereas the drifters spent time in regions of good and poor coverage and geometry. Using both sources of in situ data gives us the opportunity to compare the OI and UWLS algorithms across ranges of coverage and geometric quality within the CODAR domain. Both the OI and the UWLS algorithms had similar skill in areas of good system geometry and consistent coverage with RMS differences of $8 \mathrm{~cm} / \mathrm{s}$ and $R^{2}$ of 0.7 and provided consistent coverage on the order of $94 \%$. However, in regions of inconsistent coverage like the offshore edge of the CODAR domain, the OI improved coverage from $53 \%$ to $65 \%$ over the UWLS method while only increasing

\section{TABLE 4}

Summary table of ADCP and drifter comparison with the Optimal Interpolation (OI) and UWLS vector combining method.

\begin{tabular}{l|c|c|c|c}
\hline & \multicolumn{2}{|l|}{ ADCP Comparison } & \multicolumn{2}{c}{ Drifter Comparison } \\
\hline & UWLS & 0I & UWLS & 0I \\
\hline Temporal Coverage (\%) & 93 & 95 & 53 & 65 \\
\hline RMS u (cm/s) & 8.3 & 8.7 & 7.4 & 8.4 \\
\hline RMS v (cm/s) & 7.9 & 7.5 & 9.8 & 11.8 \\
\hline$R^{2} \mathrm{u}$ & 0.75 & 0.73 & 0.83 & 0.81 \\
\hline$R^{2} \mathrm{v}$ & 0.63 & 0.65 & 0.58 & 0.44 \\
\hline
\end{tabular}


the uncertainty approximately $1-2 \mathrm{~cm} / \mathrm{s}$ (RMS). On the basis of these results and the criteria set by the Coast Guard for consistent coverage with known uncertainties, the OI algorithm was selected as the best algorithm for inclusion in the SAROPS tool.

\section{Network Applications}

HF radar has supported a variety of applications in the Mid-Atlantic Bight over the past decade. These applications include basic research on the dynamics of the coastal ocean (Kohut et al., 2004; Dzwonkowski et al., 2009; Dzwonkowski et al., 2010; Shay et al., 2008; Ullman and Codiga, 2004; Kohut et al., 2006; Gong et al., 2010; Hunter et al., 2007) to applications centered around Coast Guard Search and Rescue (O'Donnell et al., 2005; Ullman et al., 2006) and water quality associated with floatable tracking along the New Jersey Coast. Below we highlight three applications of the integrated regional network.

\subsection{Regional Results}

The Mid-Atlantic Bight 1-year average surface currents calculated from the 2009 MARCOOS data are plotted in Figure 4. The annual average flow is generally along shelf to the southwest, with mid-shelf surface current speeds in the 5 - to $10-\mathrm{cm} / \mathrm{s}$ range. Faster currents between 10 and $17.5 \mathrm{~cm} / \mathrm{s}$ are found east of Cape Cod running offshore, along the shelf break running alongshore over the central region, and along the narrower shelf of North Carolina running across isobaths into the Gulf Stream, the strongest currents observed on the southern edge. Inshore flows are generally lower than those at mid-shelf, with increased cross-shelf flow noted at the outflow

\section{FIGURE 4}

Mid-Atlantic Bight 1-year average surface currents calculated for seasonal year 2009. Only the vectors where there was $50 \%$ data coverage are plotted. (Color versions of figures available online at: http://www.ingentaconnect.com/content/mts/mtsj/2010/00000044/00000006.)

\section{MACOORA HF Radar Network Sea Surface Currents December 2008 - November, 2009}

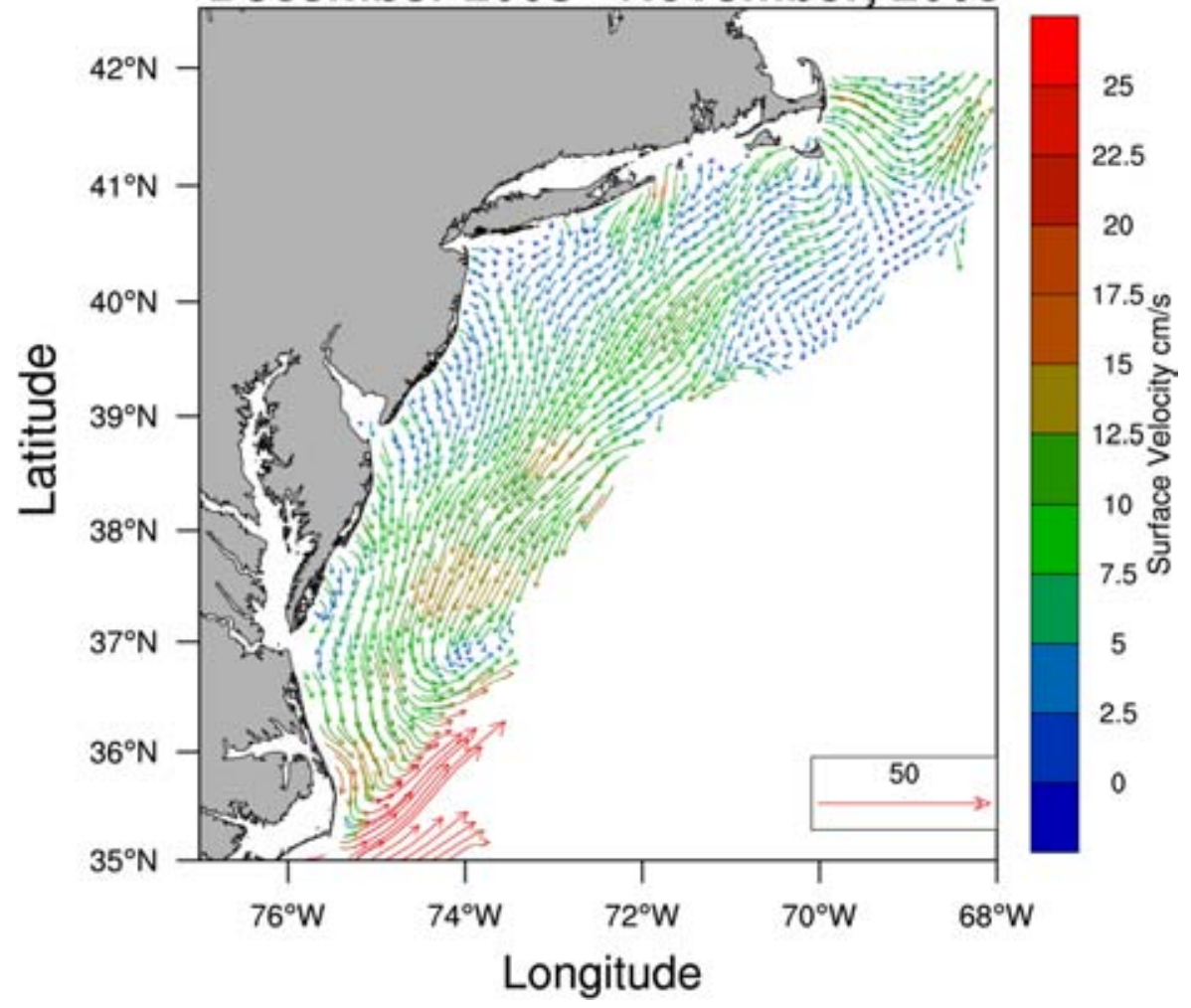

locations of the major bays that then joins the along shelf flow at the outer shelf.

Three CODAR HF Radar systems were used to study the annual and seasonal response of the New Jersey shelf currents (Gong et al., 2010). The seasonal results for the New Jersey shelf can be extended to the full MidAtlantic Bight for 1 year using the 2009 MARCOOS data set. Adopting the same seasonal definitions used by Gong et al. (2010) on the basis of their analysis of the water column stratification, winter of 2009 begins in December of 2008. As noted by Gong et al. (2010) and others, winter winds are predominately from the northwest. Average surface flow during the three winter months for the full Mid-Atlantic Bight (Figure 5a) is generally cross-shelf in the offshore direction. Stronger cross shelf flows are again observed offshore the major outflows of Long Island Sound, New York Harbor, and Delaware Bay. The MAB spring currents (Figure 5b) are generally alongshore, with stronger alongshore currents in deeper water near the shelf break, and on the southern side of the coverage over the narrower North Carolina shelf. Currents east of Cape Cod are persistently offshore to the east. Summer currents are generally the weakest, with reduced range reflecting the lower wave environment of the summer. The inner to 


\section{FIGURE 5}

Seasonal means for surface currents in the Mid-Atlantic (a) winter, (b) spring, (c) summer, and (d) fall.
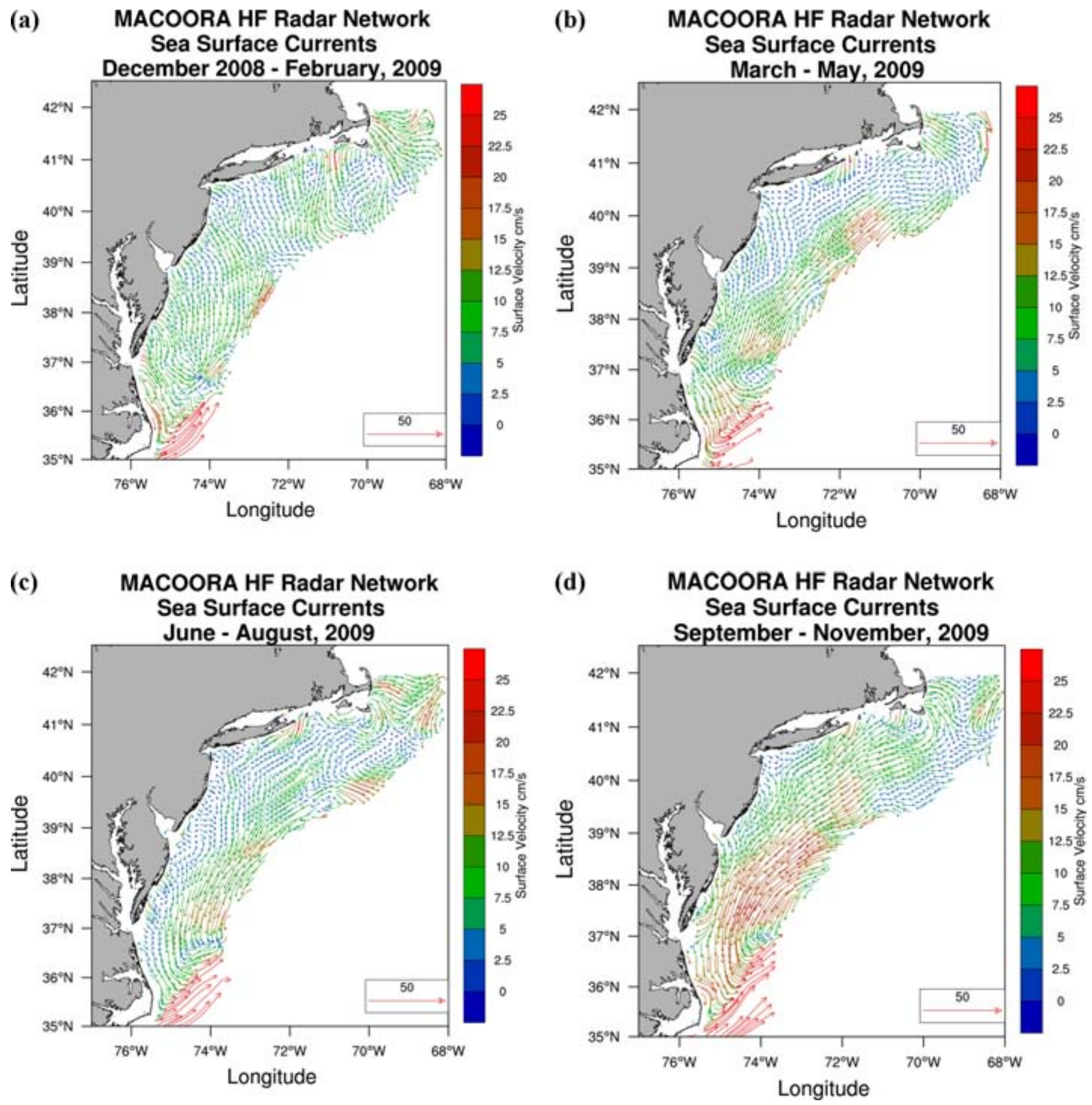

mid-shelf flows have a stronger crossshelf component than the yearly average. East of Cape Cod, some of the strongest cross-shelf flows are observed. Conversely, some of the weakest currents of the year are found on the narrow shelf east of North Carolina. As in the results of Gong et al. (2010) for the New Jersey shelf, offshore crossshelf flows are more common in the summer and winter months over most of the Mid-Atlantic Bight. Fall has the strongest surface currents, mostly along shelf and with increasing intensity as flow heads south. Except in the Bight Apex offshore Long Island and New Jersey, the alongshore flow extends across the entire shelf. This is 
the only season where the alongshore response is not blocked by an offshore flow on the inner shelf. This has important implications for the fall season and fish larvae.

\subsection{U.S. Coast Guard Search and Rescue}

MACOORA has five regional priorities supported by the 10 MARCOOS regional observing and forecasting capabilities. MACOORA's first regional priority is supporting Safety at Sea by providing improved data sets and forecast models for the U.S. Coast Guard's operational SAROPS. One primary function of SAROPS is to predict the trajectories of a large cloud (typically 5000) of simulated drifters using real-time surface current data sets and forecasts accessed via the EDS and a random flight dispersion model with pre-calculated coefficients to simulate dispersion. The random flight model coefficients, a standard deviation and half-life time scale, are precalculated on the basis of comparisons of the various surface current products with actual SLDMBs trajectories. On the basis of these historical comparisons, various current products are designated as high confidence, with a standard deviation of 0.22 knots or low confidence, with a standard deviation of 0.37 knots. In each of these standard designators, the half life time scale is set at $264 \mathrm{~min}$. New validation case studies are then generated each time a new SLDMB is deployed.

One case study was created to analyze the impact of HF radar data on the efficacy of SAROPS. The actual path of an SLDMB over 4 days (July 22, 2009-July 26, 2009) was compared with predicted path of the buoy using four data sources (STPS, NCOM, HYCOM, and HF Radar). The pre-

\section{FIGURE 6}

Screen shot of the SAROPS user interface showing the predicted dispersion of the 5,000 simulated drifters using the HYCOM data source (cloud) and path of SLDMB (line in lower left-hand corner of search area). Coast Guard search area is shown as the red box.

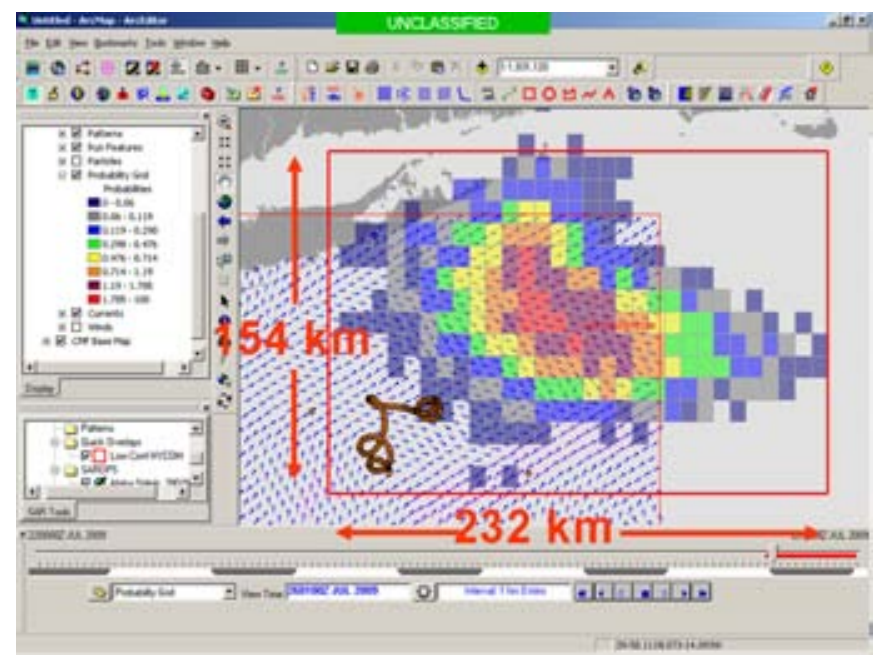

dicted dispersion of the 5000 simulated drifters using the HYCOM data source is shown in Figure 6. The search area using the HYCOM data equates to $36,000 \mathrm{~km}^{2}$. The predicted dispersion after 4 days of the 5000 simulated drifters using the HF radar data source is shown in Figure 7. The search area using the HF radar data equates to

$12,000 \mathrm{~km}^{2}$, a marked improvement over the HYCOM data source. In this test case, the HF radar was shown to provide a smaller search area centered on the actual drifter location.

\subsection{Fisheries}

MACOORA's second regional priority is Ecosystem Decision Support,

\section{FIGURE 7}

Screen shot of the SAROPS user interface showing the predicted dispersion of the 5,000 simulated drifters using the HF radar data source (cloud) and path of SLDMB (line in center of the cloud). Coast Guard search area is shown as the blue box.

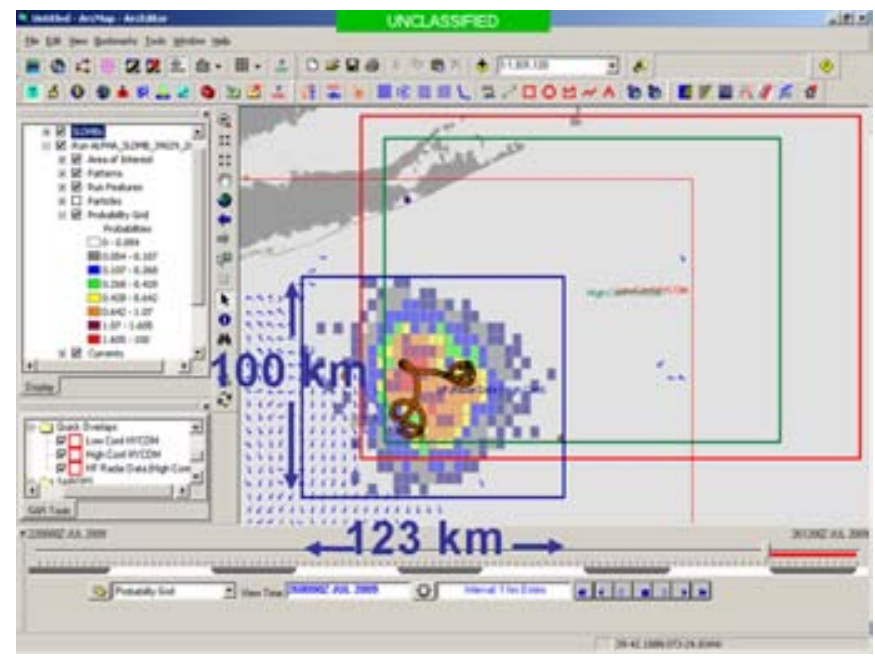


initially focusing on applications to fisheries. An application of the MAB HF Radar network is to study the larval dispersal pattern of key marine species such as summer flounder (Paralichthys dentatus). Adult summer flounders spawn during fall, winter, or spring on the shelf when temperature is between $12^{\circ} \mathrm{C}$ and $19^{\circ} \mathrm{C}$ (Smith, 1973). Survey of the monthly abundance of summer flounder eggs from 1978 to 1987 showed that the peak spawning season of $P$. dentatus is in the autumn (Packer et al., 1999). Three regions of high egg concentrations are identified ranging from Georges Bank down to Cape Hatteras. The northern population, residing mostly north of the Hudson Shelf Valley, spawns in October. The central population, near the Hudson Shelf Valley, and the southern population, south of Delaware Bay, spawns about a month later (Packer et al., 1999). The early life history stages of $P$. dentatus are pelagic, and the com- bined egg and early larvae stages are temperature dependent and can last 2 weeks (Manderson, personal communication). During this time, their movement is very limited, and dispersal is likely mainly driven by the prevailing ocean currents. On the basis of these facts, a CODAR-based virtual drifter experiment is performed on the MAB for fall 2009. Three groups of virtual drifters were deployed at the known $P$. dentatus spawning grounds during the month of October 2009. These drifters were deployed twice daily, and each group is tracked for up to 10 weeks. When a drifter reached the edge of the CODAR coverage, it was stopped, and the position was marked (Figure 8). The drifter advection algorithm includes the same random flight dispersion algorithm (Ullman et al., 2006) as used in SAROPS.

The drifter study provides us the following scientific results:

1. Surface transport in autumn is mainly downshelf.
2. Despite significant offshore loss, shoreward transport toward the major estuaries is observed. Study of Gong et al. (2010) on the central MAB showed that this only happens in the autumn season.

3. The end locations for all three spawning locations are remarkably similar, suggesting population connectivity linking the three spawning grounds.

4. The time scale of drifter transport is on the order 3-5 weeks, consistent with the time scale of the early life stages of P. dentate.

The drifter study also illustrates the MACOORA development strategy. MACOORA, through its user meetings, has identified five regional priorities: (1) safety at sea, (2) ecosystem decision support, (3) water quality, (4) coastal inundation, and (5) energy. MARCOOS is the operating arm of MACOORA, responsible for installing and maintaining the 10 regional observing capabilities. Once an operational

\section{FIGURE 8}

Virtual drifter study showing the release points (green circles), path (blue lines), and exit point (red triangles) for known Paralichthys dentatus spawning grounds during the month of October, 2009, in the (a) northern, (b) central, and (c) southern sections of the MARCO0S domain. (Color versions of figures available online at: http://www.ingentaconnect.com/content/mts/mtsj/2010/00000044/00000006.)

(a)

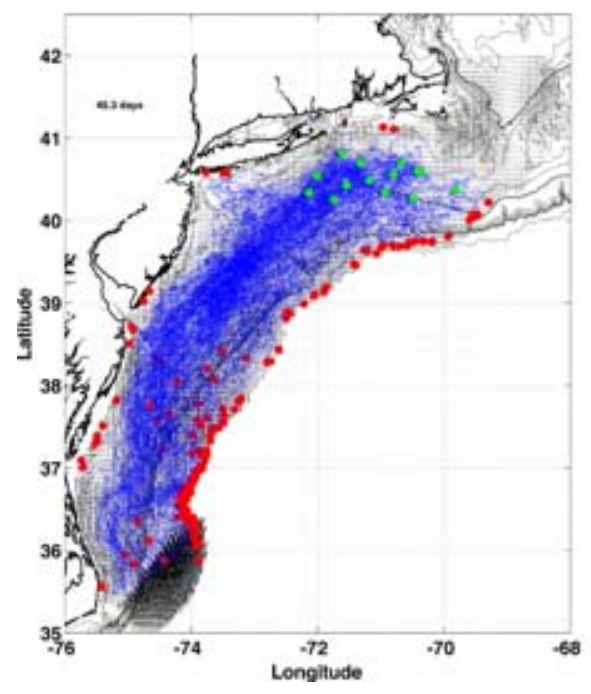

(b)

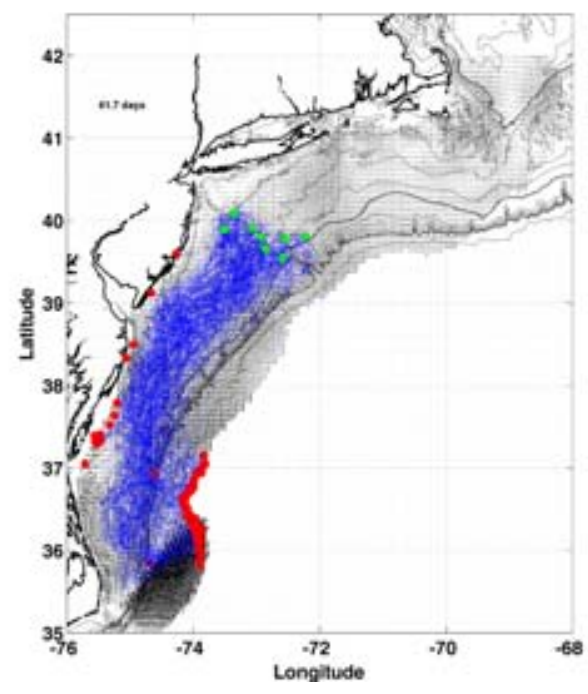

(c)

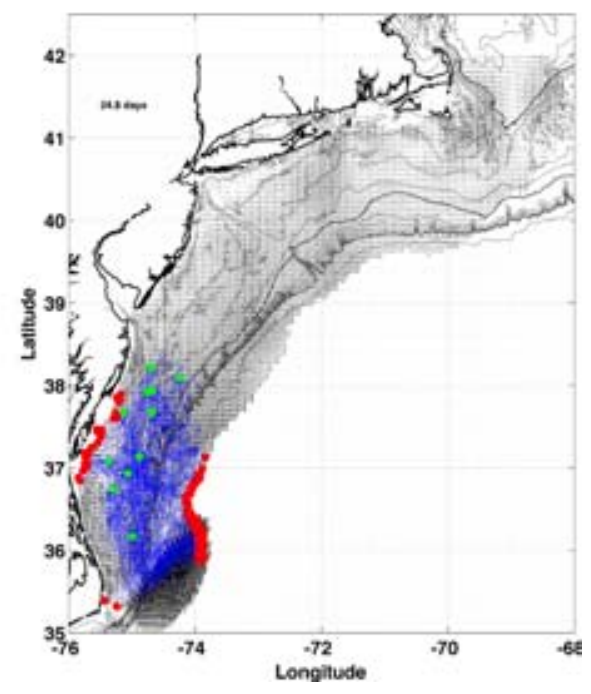


capability is developed and validated for one of the regional priorities, that capability is then available for application to the other four priorities.

\section{Conclusions}

The evolution of HF radar in the Mid-Atlantic bight has progressed from distinct subregional systems to an integrated regional network. The application of this network ranges from basic research to support of operational search and rescue. This range of application has required a very systematic approach to operation and maintenance of the hardware and software. Through this approach, we have defined QA and control measures to ensure that quality data with consistent coverage is delivered to the user groups working in the region. The approach has relied heavily on regional partners and distributed expertise working through a coordinated center.

HF radar networks like that in the Mid-Atlantic are being constructed around the country with highresolution standard-range systems nested within lower-resolution, longrange systems. With Integrated Ocean Observing System support, these regional networks are part of a coordinated national network. As we move toward products in support of national applications, like the Coast Guard Search and Rescue, there is a need for coordination and communication of all regional and subregional groups. Through a national system, lessons learned from the Mid-Atlantic and other regions around the country can drive a national resource that can support a variety of applications, as it has done in the Mid-Atlantic for the past decade.
Lead Author:

Hugh Roarty

Coastal Ocean Observation

Laboratory, Rutgers University

Email: hroarty@marine.rutgers.edu

\section{References}

Barrick, D.E., Evens, M.W., Weber, B.L. 1977. Ocean surface currents mapped

by radar. Science. 198:138-144.

doi:10.1126/science.198.4313.138.

Barrick, D.E., Lilleboe, P.M., Teague, C.C. 2001. Multi-station HF FMCW radar frequency sharing with GPS time modulation multiplexing, US Patent SN 10/109, 769.

Barrick, D.E., Lipa, B.J. 1986. Correcting for distorted antenna patterns in CODAR ocean surface measurements. IEEE J

Ocean Eng. OE-11:304-309.

doi:10.1109/JOE.1986.1145158.

Chapman, R.D., Graber, H.C. 1997. Validation of HF radar measurements. Oceanography. 10:76-79.

Crombie, D.D. 1955. Doppler spectrum of sea echo at $13.56 \mathrm{Mc}$./s. Nature. 175:681-682. doi:10.1038/175681a0.

Dzwonkowski, B., Kohut, J.T., Yan, X.-H. 2009. Seasonal differences in wind-driven across-shelf forcing and response relationships in the shelf surface layer of the central Mid-Atlantic Bight. J Geophys Res. 114(C8): C08018. doi:10.1029/2008JC004888.

Dzwonkowski, B., Lipphardt, B.L., Jr, Kohut, J.T., Yan, X.-H., Garvine, R.W. 2010. Synoptic measurements of episodic offshore flow events in the central Mid-Atlantic Bight. Cont Shelf Res. 30(12):1373-1386. doi:10.1016/j.csr.2010.05.004.

Gong, D., Kohut, J.T., Glenn, S.M. 2010. Seasonal climatology of wind-driven circulation on the New Jersey Shelf. J Geophys

Res. 115(C4):C04006. doi:10.1029/ 2009JC005520.

Hunter, E., Chant, R., Bowers, L., Glenn, S., Kohut, J. 2007. Spatial and temporal vari- ability of diurnal wind forcing in the coastal ocean. Geophys Res Lett. 34(3):L03607. doi:10.1029/2006GL028945.

Kim, S.Y., Terrill, E.J., Cornuelle, B.D. 2008. Mapping surface currents from HF radar radial velocity measurements using optimal interpolation. J Geophys Res. 113(C10): C10023. doi:10.1029/2007JC004244.

Kohut, J. 2008. MARCOOS HF Radar Quality Control/Quality Assurance Document. Available from: http:// www.marcoos.us/downloads/data/ MARCOOSHFRadarQualityControl_ 04182008.pdf.

Kohut, J., Roarty, H.J., Rivera Lemus, E. 2009. Optimizing HF radar for SAR using USCG surface drifters and moored ADCPs. The 4th Radiowave Operators Working Group (ROWG) Meeting, Norfolk, VA.

Kohut, J.T., Glenn, S.M. 2003. Improving HF radar surface current measurements with measured antenna beam patterns. J Atmos Oceanic Tech. 20(9):1303-1316. doi:10.1175/ 1520-0426(2003)020<1303:IHRSCM>2.0. $\mathrm{CO} ; 2$.

Kohut, J.T., Glenn, S.M., Chant, R.J. 2004. Seasonal current variability on the New Jersey inner shelf. J Geophys Res. 109(C7):C07S07. doi:10.1029/2003JC001963.

Kohut, J.T., Randall-Goodwin, E., Roarty, H.J., Glenn, S.M. in preparation. Evaluation of an optimal interpolation technique for combining HF radar radial vectors to totals. J Atmos Oceanic Tech.

Kohut, J.T., Roarty, H.J., Glenn, S.M. 2006. Characterizing observed environmental variability with HF Doppler radar surface current mappers and acoustic Doppler current profilers: environmental variability in the coastal ocean. IEEE J Ocean Eng. 31(4):876-884. doi:10.1109/JOE.2006.886095.

Lipa, B.J., Barrick, D.E. 1983. Least-squares methods for the extraction of surface currents from CODAR cross-loop data: application at ARSLOE. IEEE J Ocean Eng. OE-8: 226-253. doi:10.1109/JOE.1983.1145578. 
Lipa, B.J., Nyden, B., Ullman, D.S., Terrill, E.J. 2006. SeaSonde Radial Velocities: derivation and internal consistency. IEEE

J Ocean Eng. 31(4):850-861. doi:10.1109/ JOE.2006.886104.

Liu, Y., Weisberg, R.H., Merz, C.R., Lichtenwalner, S., Kirkpatrick, G.J. 2010. HF radar performance in a low energy environment: CODAR SeaSonde experience on the West Florida shelf. J Atmos Oceanic Tech. 27(10):1689-1710.

O’Donnell, J., Ullman, D.S., Spaulding, M., Howlett, E., Fake, T., Hall, P., Isaji, T., Edwards, C., Anderson, E., McClay, T., Kohut, J.T., Allen, A., Lester, S.,

Lewandowski, M. 2005. Integration of Coastal Ocean Dynamics Application Radar (CODAR) and Short-Term Predictive System (STPS) Surface Current Estimates into the Search and Rescue Optimal Planning System (SAROPS). New London, CT: U.S. Coast Guard Research \& Development Center.

Packer, D.B., Griesbach, S.J., Berrien, P.L., Zetlin, C.A., Johnson, D.L., Morse, W.W. 1999. Summer Flounder, Paralichthys dentatus, Life History and Habitat Characteristics. Washington, DC: NOAA.

Roarty, H.J. 2009. Standard Operating Procedure for Adding a New Long Range (5 MHz) HF Radar Site to the Mid-Atlantic Regional Coastal Ocean Observing System (MARCOOS). Available from: http://www. marcoos.us/downloads/data/SOP_

MARCOOS_Long_Range_Radar.doc.

Shay, L.K., Seim, H., Savidge, D., Styles, R., Weisberg, R.H. 2008. High frequency radar observing systems in SEACOOS. Mar Technol Soc J. 42(3):55-67. doi:10.4031/002533208786842435.

Smith, W.G. 1973. The distribution of summer flounder, Paralichthys dentatus, eggs and larvae on the continental shelf between Cape Cod and Cape Lookout. Fish Bull. 71(2):527-48.

Ullman, D.S., Codiga, D.L. 2004. Seasonal variation of a coastal jet in the Long Island Sound outflow region based on HF radar and Doppler current observations.

J Geophys Res. 109(C7):C07S06. doi:10.1029/2002JC001660.

Ullman, D.S., O’Donnell, J., Kohut, J., Fake, T., Allen, A. 2006. Trajectory prediction using HF radar surface currents: Monte Carlo simulations of prediction uncertainties. J Geophys Res. 111(C12):C12005. doi:10.1029/2006JC003715. 